\title{
A Robust Multi-Sensor PHD Filter Based on Multi-Sensor Measurement Clustering
}

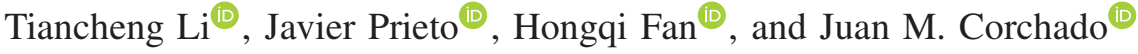

\begin{abstract}
This letter presents a novel multi-sensor probability hypothesis density (PHD) filter for multi-target tracking by means of multiple or even massive sensors that are linked by a fusion center or by a peer-to-peer network. As a challenge, we find there is little known about the statistical properties of the sensors in terms of their measurement noise, clutter, target detection probability, and even potential cross-correlation. Our approach converts the collection of the measurements of different sensors to a set of proxy and homologous measurements. These synthetic measurements overcome the problems of false and missing data and of unknown statistics, and facilitate linear PHD updating that amounts to the standard PHD filtering with no false and missing data. Simulation has demonstrated the advantages and limitations of our approach in comparison with the cuttingedge multi-sensor/distributed PHD filters.
\end{abstract}

Index Terms-PHD filtering, target tracking, sensor network.

\section{INTRODUCTION}

$\mathbf{T}$ HE PROBABILITY HYPOTHESIS DENSITY (PHD)

filter [1] has emerged as a promising approach to multitarget tracking in the presence of false and missing data. Nonetheless, the standard PHD filter presumes a single sensor [2], [3]. Exact implementation of the multi-sensor PHD filter is intractable in computation and typically, one has to resort to simplifying approximations [1]-[4]. One common alternative is known as the iterated-corrector (IC) PHD filter [2], which iterates the PHD updating step by applying the measurements of different sensors in sequence. This, however, is sensitive to the applying order of the sensors and the final result is severely dominated by the quality of the last sensor. This problem can be mitigated by the approximate product multi-sensor PHD filter [3], which, however, is computationally costly and suffers from a new problem called scale unbalance [5] that worsens as the number of sensors increases.

Alternately and especially in the case of a peer-to-peer sensor network, a bank of single sensor PHD filters may be employed, each of which uses only the measurements of one sensor, and their filtering posteriors-the PHDs-are fused for "average consensus (AC)" [6]. To this end, the average can be defined as the arithmetic average [7]-[9] or the geometric

Manuscript received June 28, 2018; accepted July 23, 2018. Date of publication August 6, 2018; date of current version October 8, 2018. This work is supported in part by the Marie Skłodowska-Curie Individual Fellowship under Grant 709267 and in part by the Spanish Ministry of Economy and Competitiveness under Torres Quevedo Grant PTQ-14-06928. The associate editor coordinating the review of this paper and approving it for publication was J. Choi. (Corresponding author: Tiancheng Li.)

T. Li is with BISITE Digital Innovation Hub, University of Salamanca, 37007 Salamanca, Spain (e-mail: t.c.li@usal.es).

J. Prieto is with BISITE Digital Innovation Hub, University of Salamanca, 37007 Salamanca, Spain, and also with the Research and Development Department, StageMotion, 34004 Palencia, Spain (e-mail: javierp@usal.es).

H. Fan is with the ATR Laboratory, National University of Defense Technology, Changsha 410073, China (e-mail: fanhongqi@nudt.edu.cn).

J. M. Corchado is with BISITE Digital Innovation Hub, University of Salamanca, 37007 Salamanca, Spain, and also with the Department of EIC, Osaka Institute of Technology, Osaka 535-8585, Japan (e-mail: corchado@usal.es).

Digital Object Identifier 10.1109/LCOMM.2018.2863387 average [10], [11], respectively. The latter coincides with the covariance intersection approach [12]. Both approaches have demonstrated superiority in terms of the filtering accuracy and/or robustness, but also various deficiencies [2], [8], [13].

Another profound challenge to the multi-sensor application is raised from the lack of the statistical knowledge of the sensors. Ingenious efforts have been made in this regard for robust filtering in the presence of unknown clutter rate and detection probability [14]-[16], unknown noise [17], etc. as well as for conservative fusion in the presence of unknown correlation between sensors [12], [18], [19].

In this letter, we consider the joint use of multiple or even massive sensors that are linked by a fusion center [19] or by a peer-to-peer network [6] and that synchronously observe the same set of targets in the presence of noises, clutter and mis-detection which are of little known statistics. The key contribution of this work is a multi-sensor measurement preprocessing procedure prior to the PHD updating step, which converts the measurements of different sensors into a set of proxy, homologous measurements. These synthetic measurements allow for linear PHD updating based on the naive Bayesian rule, and overcome the problems of misdetection, clutter and poor knowledge about the sensor statistics.

In what follows, key notations are summarized immediately. Preliminaries are introduced in Section II. The new PHD updater is devised in Section III. Simulation results are given in Section IV for comparing our approach with the centralized IC and distributed AC based PHD filters.

Notations: At time $k$, the collection of target states $\mathbf{x}_{k}^{(n)} \in$ $\mathbb{R}^{d}, n=1,2, \ldots$ is modeled by a random finite set (RFS) $X_{k}=\left\{\mathbf{x}_{k}^{(1)}, \mathbf{x}_{k}^{(2)}, \ldots, \mathbf{x}_{k}^{\left(T_{k}\right)}\right\}$ where $T_{k}$ indicates the number of targets. The states of the newborn targets are modeled by a Poisson RFS with intensity function $\gamma_{k}\left(\mathbf{x}_{k}\right)$.

We denote by $\mathcal{S}$ the set of all concerning sensors and by $\mathcal{Z}_{s, k}$ the measurement RFS of sensor $s \in \mathcal{S}$ at time $k$. The detection probability of a target with state $\mathbf{x}_{k}$ is denoted as $p_{s, k}^{\mathrm{D}}\left(\mathbf{x}_{k}\right)$ and the likelihood function of measurement $\mathbf{z}_{k} \in$ $\mathcal{Z}_{s, k}$ conditioned on state $\mathbf{x}_{k}$ is given as $g_{s, k}\left(\mathbf{z}_{k} \mid \mathbf{x}_{k}\right)$. The targets generate measurements independently across sensors and for each sensor, one target generates no more than one measurement at each sensing time. The clutter is modeled by a Poisson RFS with intensity function $\kappa_{s, k}\left(\mathbf{z}_{k}\right)$.

Given the measurement sequences at sensor $s, \mathcal{Z}_{s, 1: k} \triangleq$ $\left\{\mathcal{Z}_{s, 1}, \cdots, \mathcal{Z}_{s, k}\right\}$, we further denote by $D_{k \mid k-1}\left(\mathbf{x} \mid \mathcal{Z}_{s, 1: k-1}\right)$ and $D_{k \mid k}\left(\mathbf{x} \mid \mathcal{Z}_{s, 1: k}\right)$ the PHDs of the prior and posterior point processes $X_{k} \mid \mathcal{Z}_{s, 1: k-1}$ and $X_{k} \mid \mathcal{Z}_{s, 1: k}$ [1], respectively.

\section{PRELiminARIES}

\section{A. Single Sensor PHD Updater}

At sensor $s$, the single sensor PHD updater [1] is given by:

$$
D_{k \mid k}\left(\mathbf{x} \mid \mathcal{Z}_{s, 1: k}\right)=L_{\mathcal{Z}_{s, k}}(\mathbf{x}) D_{k \mid k-1}\left(\mathbf{x} \mid \mathcal{Z}_{s, 1: k-1}\right)
$$


where the pseudo-likelihood $L_{\mathcal{Z}_{s, k}}(\mathbf{x})$ can be decomposed as $L_{\mathcal{Z}_{s, k}}(\mathbf{x})=1-p_{s, k}^{\mathrm{D}}(\mathbf{x})+\sum_{\mathbf{z} \in \mathcal{Z}_{s, k}} L_{\mathbf{z}}(\mathbf{x})$ with

$$
L_{\mathbf{z}}(\mathbf{x}) \triangleq \frac{p_{s, k}^{\mathrm{D}}(\mathbf{x}) g_{s, k}(\mathbf{z} \mid \mathbf{x})}{\kappa_{s, k}(\mathbf{z})+G_{s, k}} .
$$

Here, $G_{s, k} \triangleq \int p_{s, k}^{\mathrm{D}}(\mathbf{x}) g_{s, k}(\mathbf{z} \mid \mathbf{x}) D_{k \mid k-1}\left(\mathbf{x} \mid \mathcal{Z}_{s, 1: k-1}\right) d \mathbf{x}$.

The integral of the PHD over a region $\mathcal{R} \subseteq \mathbb{R}^{d}$ gives the expected number of targets present in $\mathcal{R}$, i.e., $\int_{\mathcal{R}} D_{k \mid k}\left(\mathbf{x} \mid \mathcal{Z}_{s, 1: k}\right) d \mathbf{x}=\mathrm{E}\left[\left|X_{k} \cap \mathcal{R}\right|\right]$.

\section{B. Multi-Sensor Measurement Collection}

Basically, there are two types of sensor network topologies, one having a fusion center that is linked with all sensors [19] and the other being a peer-to-peer network where each sensor is only linked with its neighbors [6]. In the former case, all sensors transmit their data directly to the fusion center while in the latter, iterative flooding communications [20] need to be applied between neighbors for data dissemination over the sensor network and then each sensor serves equivalently as a fusion center. We note that, if the number of communication iterations $t_{\mathrm{F}}<D_{m}$ where $D_{m}$ is the network diameter [6], each sensor will receive sensor data only from sensors within its $t_{\mathrm{F}}$ neighborhood by flooding. One more comment is in order. To reduce the communication and local memory requirement, bandwidth reduction such as via compressive sensing and quantization [21] may be applied. Hereafter, we assume sensor $s$ having received the measurements of multiple sensors and we leave the communication issues.

\section{Novel Multi-Sensor PHD Updater}

This section presents a multi-sensor measurementpreprocessing procedure in four steps which leads to a novel multi-sensor PHD updater.

\section{A. Step 1: Project Measurements to State Space}

We consider the common nonlinear range-bearing measurement model given as $\mathbf{z}_{s, k}=\left[r_{s, k}, \theta_{s, k}\right]^{\mathrm{T}}$ with

$$
\left[\begin{array}{c}
r_{s, k} \\
\theta_{s, k}
\end{array}\right]=\left[\begin{array}{c}
\sqrt{\left(p_{x, k}-x_{s}\right)^{2}+\left(p_{y, k}-y_{s}\right)^{2}} \\
\arctan \left(\frac{p_{y, k}-y_{s}}{p_{x, k}-x_{s}}\right)
\end{array}\right]+\left[\begin{array}{c}
v_{s, r} \\
v_{s, \theta}
\end{array}\right],
$$

where $\left[p_{x, k}, p_{y, k}\right]^{\mathrm{T}}$ and $\left[x_{s}, y_{s}\right]^{\mathrm{T}}$ are the positions of the underlying target and sensor $s$, respectively while $v_{s, r}$ and $v_{s, \theta}$ are white noises affecting range $r_{s, k}$ and bearing $\theta_{s, k}$, respectively, which are not limited to being Gaussian-distributed.

The projection of $\left[r_{s, k}, \theta_{s, k}\right]^{\mathrm{T}}$ on the state space yields a position-detection $\mathbf{y}_{s, k}=\left[\hat{p}_{x, k}, \hat{p}_{y, k}\right]^{\mathrm{T}}$ with

$$
\begin{aligned}
& \hat{p}_{x, k}=\operatorname{sgn}_{x} b_{s, k}+x_{s}, \\
& \hat{p}_{y, k}=\operatorname{sgn}_{y} \tan \left(\left|\theta_{s, k}-\bar{v}_{s, \theta}\right|\right) b_{s, k}+y_{s},
\end{aligned}
$$

where $b_{s, k} \triangleq \sqrt{\frac{\left(r_{s, k}-\bar{v}_{s, r}\right)^{2}}{1+\left(\tan \left(\left|\theta_{s, k}-\bar{v}_{s, \theta}\right|\right)\right)^{2}}}, \bar{v}_{s, r}$ and $\bar{v}_{s, \theta}$ are the statistical means of $v_{s, r}$ and $v_{s, \theta}$, respectively, and the signs $\operatorname{sgn}_{x}$ and $\operatorname{sgn}_{y}$ rely on the quadrant of the underlying target in the sensing range of the sensor, i.e., $\operatorname{sgn}_{x}=1, \operatorname{sgn}_{y}=1$ when $\theta_{s, k} \in(0, \pi / 2], \operatorname{sgn}_{x}=-1, \operatorname{sgn}_{y}=1$ when $\theta_{s, k} \in(\pi / 2, \pi]$, $\operatorname{sgn}_{x}=1, \operatorname{sgn}_{y}=-1$ when $\theta_{s, k} \in(-\pi / 2,0]$ and $\operatorname{sgn}_{x}=$ $-1, \operatorname{sgn}_{y}=-1$ when $\theta_{s, k} \in(-\pi,-\pi / 2]$.
We note that the nonlinear conversions (4) and (5) are statistically biased [22]. When higher order statistical information about the noise is available, such as the variance, a debiasing scheme should be applied [22] to account for the bias.

\section{B. Step 2: Cluster Multi-Sensor Data}

At this step, we apply the constrained, hierarchical clustering scheme [23] to the converted measurements. That is, the density criterion is employed to group the raw data (for which the number of clusters is automatically determined) and the distance criterion to further divide the overlapped clusters (due to close-distributed targets). As the end of this step, data corresponding to the same target are expected to be grouped into one cluster. The shape and size of the cluster just indicate the uncertainty of the measurements of that target.

\section{Step 3: Generate Proxy Measurements and Likelihood}

Denote by $C_{s, k}$ the number of the clusters obtained at sensor $s$ at time $k$, each of which indicates a potential target. For the cluster $c=1, \cdots, C_{s, k}$ of size $J_{s}^{[c]}$ whose data-points can be written as $\left\{\mathbf{y}_{j, k}^{[c]}\right\}_{j=1, \cdots, J_{s}^{[c]}}$, its centroid $\overline{\mathbf{y}}_{s, k}^{[c]}$ is given as

$$
\overline{\mathbf{y}}_{s, k}^{[c]}=\frac{1}{J_{s}^{[c]}} \sum_{j=1}^{J_{s}^{[c]}} \mathbf{y}_{j, k}^{[c]} .
$$

This provided the (position) detection of the underlying target [23], [24], but no information about the target velocity. Here, we remain using it as (position-related) measurement, i.e.,

$$
\overline{\mathbf{y}}_{s, k}^{[c]}=\left[p_{x, k}, p_{y, k}\right]^{\mathrm{T}}+\mathbf{v}_{s, k}^{[c]},
$$

where the residual $\mathbf{v}_{s, k}^{[c]}$ amounts to the proxy measurement noise, whose covariance is calculated as

$$
\Sigma_{\mathbf{v}_{s, k}^{[c]}}=\sum_{j=1}^{J_{s}^{[c]}}\left(\overline{\mathbf{y}}_{s, k}^{[c]}-\mathbf{y}_{j, k}^{[c]}\right)\left(\overline{\mathbf{y}}_{s, k}^{[c]}-\mathbf{y}_{j, k}^{[c]}\right)^{\mathrm{T}} .
$$

Denoting the target state $\mathbf{x}_{k}=\left[p_{x, k}, p_{y, k}, \dot{p}_{x, k}, \dot{p}_{y, k}\right]^{\mathrm{T}}$ consisting of the position $\left[p_{x, k}, p_{y, k}\right]^{\mathrm{T}}$ and the velocity $\left[\dot{p}_{x, k}, \dot{p}_{y, k}\right]^{\mathrm{T}}$, the measurement function (7) can be written as

$$
\overline{\mathbf{y}}_{s, k}^{[c]}=\mathbf{H}_{\mathrm{pos}} \mathbf{x}_{k}+\mathbf{v}_{s, k}^{[c]},
$$

where $\mathbf{H}_{\mathrm{pos}}=\left[\mathbf{I}_{2}, \mathbf{0}_{2}\right]$.

Simply, a Gaussian-distributed variable $\mathbf{v}_{s, k}^{[c]}$ corresponds to the proxy Gaussian likelihood function

$$
g_{k}^{\prime}\left(\overline{\mathbf{y}}_{s, k}^{[c]} \mid \mathbf{x}_{k}\right)=\mathcal{N}\left(\overline{\mathbf{y}}_{s, k}^{[c]} ; \mathbf{H}_{\mathrm{pos}} \mathbf{x}_{k}, \Sigma_{\mathbf{v}_{s, k}^{[c]}}\right),
$$

where $\mathcal{N}(\mathbf{x} ; \mathbf{m}, \Sigma)$ denotes a Gaussian function of $\mathbf{x}$ with mean $\mathbf{m}$ and covariance $\Sigma$.

\section{Step 4: Modify the PHD Updater}

Since the measurements are postulated independent across the sensors, it is unlikely for the same target to be missed in detection or for the false alarm to occur at the same area, for the majority of the concerning sensors. Therefore, the synthetic position measurements $\mathcal{C}_{s, k} \triangleq\left\{\overline{\mathbf{y}}_{s, k}^{[c]}\right\}_{c=1,2, \ldots C_{s, k}}$ are expected 
to be free of misdetection and clutter, indicating $\kappa_{s, k}(\mathbf{y})=0$ and $p_{s, k}^{\mathrm{D}}(\mathbf{x})=1$, respectively. This leads to the proxy pseudolikelihood

$$
L_{\mathcal{C}_{s, k}}(\mathbf{x}) \triangleq \sum_{\mathbf{y} \in \mathcal{C}_{s, k}} \frac{g_{k}^{\prime}(\mathbf{y} \mid \mathbf{x})}{\int g_{k}^{\prime}(\mathbf{y} \mid \mathbf{x}) D_{k \mid k-1}\left(\mathbf{x} \mid \mathcal{C}_{s, k-1}\right) d \mathbf{x}} .
$$

Substituting $\mathcal{Z}_{s, k}, L_{\mathcal{Z}_{s, k}}(\mathbf{x})$ of (1) by $\mathcal{C}_{s, k}, L_{\mathcal{C}_{s, k}}(\mathbf{x})$ in (11), respectively, yields a modified PHD updater as

$$
D_{k \mid k}\left(\mathbf{x} \mid \mathcal{C}_{s, k}\right)=\sum_{\mathbf{y} \in \mathcal{C}_{s, k}} \frac{g_{k}^{\prime}(\mathbf{y} \mid \mathbf{x}) D_{k \mid k-1}\left(\mathbf{x} \mid \mathcal{C}_{s, k-1}\right)}{\int g_{k}^{\prime}(\mathbf{y} \mid \mathbf{x}) D_{k \mid k-1}\left(\mathbf{x} \mid \mathcal{C}_{s, k-1}\right) d \mathbf{x}} .
$$

By this, the expected number of targets becomes consistent with the number of the clusters, i.e.,

$$
\mathrm{E}\left[T_{k} \mid \mathcal{C}_{s, k}\right]=\int D_{k \mid k}\left(\mathbf{x} \mid \mathcal{C}_{s, k}\right) d \mathbf{x}=\left|\mathcal{C}_{s, k}\right|=C_{s, k}
$$

\section{E. Gaussian Mixture (GM) and Particle Implementation}

The proposed PHD filter can be implemented by approximating the predicted and updated PHDs using either a Gaussian mixture [25] (GM, namely the GM-PHD filter) or a set of weighted particles [26] (namely the particle PHD filter). That is, $D_{k \mid k-1}\left(\mathbf{x} \mid \mathcal{C}_{s, k-1}\right) \approx$ $\sum_{j=1}^{J_{s, k \mid k-1}} w_{s, k \mid k-1}^{(j)} \psi_{s, k \mid k-1}^{(j)}(\mathbf{x})$ for the predicted PHD and $D_{k \mid k}\left(\mathbf{x} \mid \mathcal{C}_{s, k}\right) \approx \sum_{j=1}^{J_{s, k \mid k}} w_{s, k \mid k}^{(j)} \psi_{s, k \mid k}^{(j)}(\mathbf{x})$ for the updated PHD. Here, the weights $w_{s, k}^{(j)} \geq 0$ and the functions $\psi_{s, k}^{(j)}(\mathbf{x})$ are given by Gaussian function $\mathcal{N}\left(\mathbf{x} ; \mathbf{m}_{s, k}^{(j)}, \mathbf{P}_{s, k}^{(j)}\right)$ in the case of the GM-PHD filter and by delta function $\delta_{\mathbf{x}_{s, k}^{(j)}}(\mathbf{x})$ in the case of the particle PHD filter; $\mathbf{m}_{s, k}^{(j)}$ and $\mathbf{P}_{s, k}^{(j)}$ denote the mean and covariance the Gaussian component (GC) $j$, respectively and $\mathbf{x}_{s, k}^{(j)}$ is the state of particle $j$, at time $k$ and sensor $s$.

The key difference of our approach to the standard implementation given in [25] and [26] lies on the weight updating from $w_{s, k \mid k-1}^{(j)}$ to $w_{s, k \mid k}^{(j)}$, due to the new measurements and new likelihood function. In short, for the particle implementation, the weight of particle $j$ is updated by (cf. Eq.(22) of [25])

$$
w_{s, k \mid k}^{(j)}=\sum_{\mathbf{y} \in \mathcal{C}_{s, k}} \Omega^{-1} g_{k}^{\prime}\left(\mathbf{y} \mid \mathbf{x}_{s, k}^{(j)}\right) w_{s, k \mid k-1}^{(j)},
$$

where $\Omega=\sum_{i=1}^{J_{s, k \mid k-1}} g_{k}^{\prime}\left(\mathbf{y} \mid \mathbf{x}_{s, k}^{(j)}\right) w_{s, k \mid k-1}^{(i)}$.

For the GM implementation, each predicted GC will generate a new GC for (and only for) each measurement and so $J_{s, k \mid k}=J_{s, k \mid k-1} C_{s, k}$. For measurement $\mathbf{y} \in \mathcal{C}_{s, k}$, the weight of the new $\mathrm{GC} l=1, \cdots, J_{s, k \mid k}$ that branches from the predicted GC $j$ with mean $\mathbf{m}_{s, k \mid k-1}^{(j)}$ and covariance $\mathbf{P}_{s, k \mid k-1}^{(j)}$ is (cf. Eq. (34) of [26])

$$
w_{s, k \mid k}^{(l)} \triangleq w_{s, k}^{(j)}(\mathbf{y})=\Omega^{\prime-1} q_{s, k}^{(j)}(\mathbf{y}) w_{s, k \mid k-1}^{(j)},
$$

where $\Omega^{\prime}=\sum_{i=1}^{J_{s, k \mid k-1}} q_{s, k}^{(i)}(\mathbf{y}) w_{s, k \mid k-1}^{(i)}$, and

$q_{s, k}^{(j)}(\mathbf{y}) \triangleq \mathcal{N}\left(\mathbf{y} ; \mathbf{H}_{\mathrm{pos}} \mathbf{m}_{s, k \mid k-1}^{(j)}, \mathbf{H}_{\mathrm{pos}} \mathbf{P}_{s, k \mid k-1}^{(j)} \mathbf{H}_{\mathrm{pos}}^{\mathrm{T}}+\Sigma_{\mathbf{v}_{s, k}^{[c]}}\right)$.

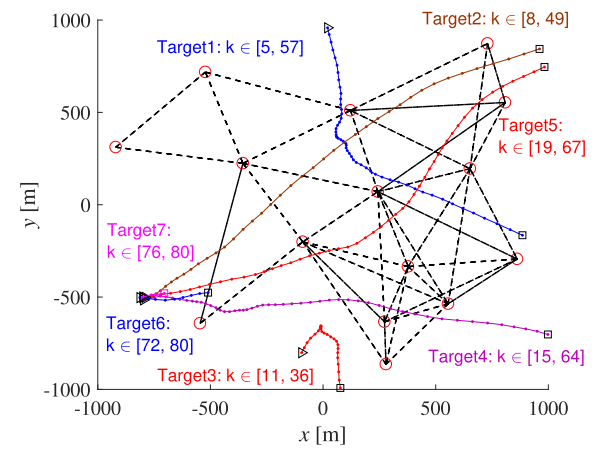

Fig. 1. ROI involving 15 sensors and 7 targets. The communication links between neighbor sensors (located at red ' $\circ$ ') are shown in black ashed lines and the target trajectories in colored lines with dots. Each target starts at ' $\triangle$ ' and ends at ' $\square$ ' with persisting time noted.

\section{Simulation Results}

In the simulation, the proposed GM-PHD filter was compared with three cutting-edge multi-sensor PHD filters: the unscented transformation based GM (UGM)-PHD filter and the particle PHD filter based on the distributed AC for posterior PHD averaging [8], [9], and the centralized IC-based [2] UGM-PHD filer. In addition, a noncooperative filter in which each local UGM-PHD filter ran independently of the others was also implemented.

The concerning region of interest (ROI) was a planar region $[-1000 \mathrm{~m}, 1000 \mathrm{~m}] \times[-1000 \mathrm{~m}, 1000 \mathrm{~m}]$ that was synchronously monitored by 15 sensors as shown in Fig. 1 . The sensor network diameter was $D_{m}=3$. In our simulation, different numbers of flooding iterations [20], from $t=0$ (when each sensor did not interact with the others) to $t=3$, were performed between neighboring sensors for disseminating measurements (in the IC-based approach [2] and our approach) or the GM parameters (in the AC-based approaches [8], [9]).

At each sensing time $k$, the newborn target intensity function was $\gamma_{k}(\mathbf{x})=\sum_{i=1}^{3} \lambda_{i} \mathcal{N}\left(\mathbf{x} ; \mathbf{m}_{i}, \mathbf{P}_{\gamma}\right)$, with $\lambda_{1}=\lambda_{2}=\lambda_{3}=0.05, \mathbf{m}_{1}=[0 \mathrm{~m}, 950 \mathrm{~m}, 0 \mathrm{~m} / \mathrm{s}$, $-30 \mathrm{~m} / \mathrm{s}]^{\mathrm{T}}, \quad \mathbf{m}_{2}=[-100 \mathrm{~m},-800 \mathrm{~m}, \quad 10 \mathrm{~m} / \mathrm{s}, \quad 30 \mathrm{~m} / \mathrm{s}]^{\mathrm{T}}$, $\mathbf{m}_{3}=[-800 \mathrm{~m},-500 \mathrm{~m}, 20 \mathrm{~m} / \mathrm{s}, 0 \mathrm{~m} / \mathrm{s}]^{\mathrm{T}}$, and $\mathbf{P}_{\gamma}=$ $\operatorname{diag}\left(\left[100 \mathrm{~m}^{2}, 100 \mathrm{~m}^{2}, 25 \mathrm{~m}^{2} / \mathrm{s}^{2}, 25 \mathrm{~m}^{2} / \mathrm{s}^{2}\right]^{\mathrm{T}}\right)$. The target survival probability from time $k-1$ to time $k$ was 0.98 and the survival target followed a nearly constant velocity motion as $\mathbf{x}_{k}=\mathbf{F}_{\mathrm{cv}} \mathbf{x}_{k-1}+\mathbf{G}_{\mathrm{cv}} \mathbf{u}_{k-1}$, where $\mathbf{F}_{\mathrm{cv}}=\left[\mathbf{I}_{2}, \mathbf{I}_{2} ; \mathbf{0}_{2}, \mathbf{I}_{2}\right]$, $\mathbf{G}_{\mathrm{cv}}=\left[0.5 \mathbf{I}_{2} ; \mathbf{I}_{2}\right]$, and $\mathbf{u}_{k} \sim \mathcal{N}\left(\mathbf{u} ; \mathbf{0}_{2} \mathrm{~m} / \mathrm{s}^{2}, 25 \mathbf{I}_{2} \mathrm{~m}^{2} / \mathrm{s}^{4}\right)$.

Each sensor had a target detection probability $p^{\mathrm{D}}\left(\mathbf{x}_{k}\right)=$ 0.95 and a sensing range that was a disc of radius $3000 \mathrm{~m}$ around the location of the sensor. The clutter was uniformly distributed on the sensor's sensing range with an average rate of 10 points per scan, indicating clutter intensity $\kappa_{s, k}\left(\mathbf{z}_{k}\right)=$ $1 /(600 \pi)$. The measurement model was given in (3) with $v_{s, r} \sim \mathcal{N}\left(v ; 0, \sigma_{s, r}^{2}\right), v_{s, \theta} \sim \mathcal{N}\left(v ; 0, \sigma_{s, \theta}^{2}\right)$, where $\sigma_{s, r}=10 \mathrm{~m}$, $\sigma_{s, \theta}=\pi / 90 \mathrm{rad}$. We reiterate that our approach did not need the exact knowledge of these sensor parameters, while they were critically important and exactly provided to the comparison filters for their best performance.

The simulation was performed for 100 runs using randomly generated measurement series in each run but the same target trajectories. Each run consisted of 80 filtering steps. The 


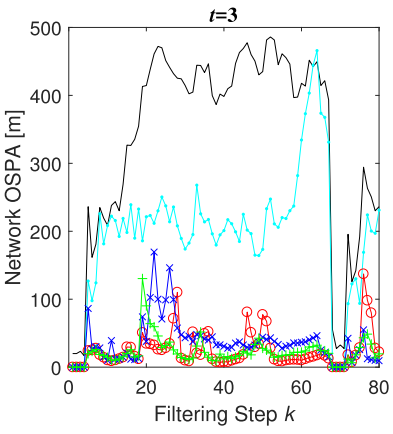

(a)

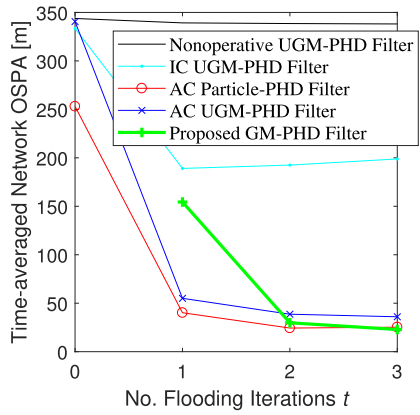

(b)
Fig. 2. OSPA. (a) Network OSPA against filtering step $k$ (when $t=3$ ) and (b) time-averaged network OSPA against the number $t$ of flooding iterations.

TABLE I

Average Computing Time for Each Filtering Step $(t=3)$

\begin{tabular}{|c||c||c||c||c||c|}
\hline Filters & Non. & IC & AC P & AC UGM & Proposed \\
\hline Time $(\mathrm{s})$ & 0.0107 & 0.6373 & 0.0186 & 0.0234 & 0.0205 \\
\hline
\end{tabular}

optimal sub-pattern assignment (OSPA) metric [27] was used with the cut-off parameter $c=1000 \mathrm{~m}$ and the order parameter $p=2$. Furthermore, we defined network OSPA as the average of OSPAs obtained by all sensors in the network at each sampling step and time-averaged network OSPA as the average of the network OSPAs over all filtering steps. The network OSPA for each filter step when $t=3$, and the time-averaged network OSPA for different $t$ s, were given in Fig. 2. When $t=3$, the average computing time of different filters for each filtering step was given in Table I. The results showed:

- All multi-sensor filters achieved better accuracy than the noncooperative, single sensor filter. The gain increased with the increase of the number of the sensors (as $t$ increased), until a certain point. However, the proposed GM-PHD filter could not work with a single sensor (and so there was no plot for $t=0$ ).

- The AC-based particle PHD filter outperformed the UGM PHD filters based on either IC or AC.

- When $t=1$, the performance of the proposed GM-PHD filter was disappointing as compared to distributed AC UGM/particle PHD filters although it still outperformed the noncooperative or IC based UGM-PHD filters.

- The proposed GM-PHD filter yielded the best accuracy of all when $t=3$.

- The proposed GM-PHD filter performed similar to the AC based particle/UGM PHD filters in computing efficiency, much faster than the IC based UGM-PHD filter.

\section{CONCLUSION}

A novel, robust multi-sensor PHD updater has been devised for dealing with the poor statistical knowledge of the sensors in tracking an unknown number of targets, which particularly appeals to the joint use of massive sensors. Future extension of our work is to consider correlated clutter, asynchronous sensing rate and limited field of view of the sensors.

\section{REFERENCES}

[1] R. P. S. Mahler, "Multitarget Bayes filtering via first-order multitarget moments," IEEE Trans. Aerosp. Electron. Syst., vol. 39, no. 4, pp. 1152-1178, Oct. 2003.
[2] R. Mahler, "The multisensor PHD filter: I. General solution via multitarget calculus," Proc. SPIE, vol. 7336, p. 73360E, May 2009.

[3] R. Mahler, "Approximate multisensor CPHD and PHD filters," in Proc 13th Int. Conf. Inf. Fusion, Edinburgh, U.K., Jul. 2010, pp. 1-8.

[4] S. Nannuru, S. Blouin, M. Coates, and M. Rabbat, "Multisensor CPHD filter," IEEE Trans. Aerosp. Electron. Syst., vol. 52, no. 4, pp. 1834-1854, Aug. 2016.

[5] C. Ouyang and H. Ji, "Scale unbalance problem in product multisensor PHD filter," Electron Lett., vol. 47, no. 22, pp. 1247-1249, Oct. 2011.

[6] R. Olfati-Saber, J. A. Fax, and R. M. Murray, "Consensus and cooperation in networked multi-agent systems," Proc. IEEE, vol. 95, no. 1, pp. 215-233, Jan. 2007.

[7] R. L. Streit, "Multisensor multitarget intensity filter," in Proc. 11th Int. Conf. Inf. Fusion, Cologne, Germany, Jun./Jul. 2008, pp. 1-8.

[8] T. Li, J. M. Corchado, and S. Sun, "Partial consensus and conservative fusion of Gaussian mixtures for distributed PHD fusion," IEEE Trans. Aerosp. Electron. Syst., to be published. [Online]. Available: https://arxiv.org/abs/1711.10783

[9] T. Li. (Dec. 2017). "Distributed SMC-PHD fusion for partial, arithmetic average consensus." [Online]. Available: https://arxiv.org/abs/1712. 06128

[10] G. Battistelli, L. Chisci, C. Fantacci, A. Farina, and A. Graziano, "Consensus CPHD filter for distributed multitarget tracking," IEEE J. Sel. Topics Signal Process., vol. 7, no. 3, pp. 508-520, Jun. 2013.

[11] M. Uney, D. E. Clark, and S. J. Julier, "Distributed fusion of PHD filters via exponential mixture densities," IEEE J. Sel. Topics Signal Process., vol. 7, no. 3, pp. 521-531, Jun. 2013.

[12] S. Julier and J. K. Uhlmann, General Decentralized Data Fusion With Covariance Intersection (CI). Boca Raton FL, USA: CRC Press, 2001, ch. 12 , pp. $12-25$.

[13] S. Mori, K.-C. Chang, and C.-Y. Chong, "Comparison of track fusion rules and track association metrics," in Proc. 15th Int. Conf. Inf. Fusion, Singapore, Jul. 2012, pp. 1996-2003.

[14] R. P. S. Mahler, B.-T. Vo, and B.-N. Vo, "CPHD filtering with unknown clutter rate and detection profile," IEEE Trans. Signal Process., vol. 59, no. 8, pp. 3497-3513, Aug. 2011.

[15] C. Li, W. Wang, T. Kirubarajan, J. Sun, and P. Lei, "PHD and CPHD filtering with unknown detection probability," IEEE Trans. Signal Process., vol. 66, no. 14, pp. 3784-3798, Jul. 2018.

[16] W. Liu, Y. Chen, H. Cui, and C. Wen, "A non-uniform clutter intensity estimation algorithm for random finite set filters," IEEE Trans. Aerosp. Electron. Syst., to be published. [Online]. Available: https://ieeexplore.ieee.org/document/8353789

[17] W. Li, Y. Jia, J. Du, and J. Zhang, "PHD filter for multi-target tracking by variational Bayesian approximation," in Proc. 52nd IEEE Conf. Decis. Control, Florence, Italy, Dec. 2013, pp. 7815-7820.

[18] J. K. Uhlmann, "Covariance consistency methods for fault-tolerant distributed data fusion," Inf. Fusion, vol. 4, no. 3, pp. 201-215, Sep. 2003.

[19] X. R. Li, Y. Zhu, J. Wang, and C. Han, "Optimal linear estimation fusion. I. Unified fusion rules," IEEE Trans. Inf. Theory, vol. 49, no. 9, pp. 2192-2208, Sep. 2003

[20] T. Li, J. M. Corchado, and J. Prieto, "Convergence of distributed flooding and its application for distributed Bayesian filtering," IEEE Trans. Signal Inf. Process. Netw., vol. 3, no. 3, pp. 580-591, Sep. 2017.

[21] P. S. Rossi, D. Ciuonzo, K. Kansanen, and T. Ekman, "On energy detection for MIMO decision fusion in wireless sensor networks over NLOS fading," IEEE Commun. Lett., vol. 19, no. 2, pp. 303-306, Feb. 2015.

[22] T. Li, J. M. Corchado, J. Bajo, S. Sun, and J. F. De Paz, "Effectiveness of Bayesian filters: An information fusion perspective," Inf. Sci., vol. 329, pp. 670-689, Feb. 2016.

[23] T. Li, F. De la Prieta, J. M. Corchado, and J. Bajo, "Multi-source homogeneous data clustering for multi-target detection from cluttered background with misdetection," Appl. Soft Comput., vol. 60, pp. 436-446, Nov. 2017.

[24] T. Li, J. Corchado, and H. Chen, "Distributed flooding-then-clustering: A lazy networking approach for distributed multiple target tracking," in Proc. 21st Int. Conf. Inf. Fusion, Cambridge, U.K., Jul. 2018, pp. 1-8.

[25] B. N. Vo and W. K. Ma, "The Gaussian mixture probability hypothesis density filter," IEEE Trans. Signal Process., vol. 54, no. 11, pp. 4091-4104, Nov. 2006.

[26] B.-N. Vo, S. Singh, and A. Doucet, "Sequential Monte Carlo methods for multitarget filtering with random finite sets," IEEE Trans. Aerosp. Electron. Syst., vol. 41, no. 4, pp. 1224-1245, Oct. 2005.

[27] D. Schuhmacher, B.-T. Vo, and B.-N. Vo, "A consistent metric for performance evaluation of multi-object filters," IEEE Trans. Signal Process., vol. 56, no. 8, pp. 3447-3457, Aug. 2008. 